\title{
FAK induces expression of Prx1 to promote tenascin-C-dependent fibroblast migration
}

\author{
David M. McKean, ${ }^{1}$ Lila Sisbarro, ${ }_{1}^{1}$ Dusko Ilic, ${ }^{3}$ Nihal Kaplan-Alburquerque ${ }^{2}$ Raphael Nemenoff, ${ }^{2}$ \\ Mary Weiser-Evans, ${ }^{1}$ Michael J. Kern, ${ }^{4}$ and Peter Lloyd Jones ${ }^{1}$ \\ ${ }^{1}$ Department of Pediatrics and ${ }^{2}$ Department of Medicine, University of Colorado Health Sciences Center, Denver, CO 80262 \\ ${ }^{3}$ Department of Stomatology, University of California, San Francisco, San Francisco, CA 94143 \\ ${ }^{4}$ Department of Cell Biology and Anatomy, Medical University of South Carolina, Charleston, SC 29425
}

F ibroblast migration depends, in part, on activation of FAK and cellular interactions with tenascin-C (TN-C). Consistent with the idea that FAK regulates TN-C, migration-defective FAK-null cells expressed reduced levels of TN-C. Furthermore, expression of FAK in FAK-null fibroblasts induced TN-C, whereas inhibition of FAK activity in FAK-wild-type cells had the opposite effect. Paired-related homeobox 1 (Prx1) encodes a homeobox transcription factor that induces $\mathrm{TN}-\mathrm{C}$ by interacting with a binding site within the TN-C promoter, and it also promotes fibroblast migration. Therefore, we hypothesized that FAK regulates TN-C by controlling the DNA-binding activity of Prx1 and/or by inducing Prx1 expression. Prx1-homeodomain binding site complex formation observed with FAK-wild-type fibroblasts failed to occur in FAK-null fibroblasts, yet expression of Prx1 in these cells induced TN-C promoter activity. Thus, FAK is not essential for Prx1 DNA-binding activity. However, activated FAK was essential for Prx1 expression. Functionally, Prx1 expression in FAK-null fibroblasts restored their ability to migrate toward fibronectin, in a manner that depends on $\mathrm{TN}-\mathrm{C}$. These results appear to be relevant in vivo because Prx1 and TN-C expression levels were reduced in FAK-null embryos. This paper suggests a model whereby FAK induces Prx 1, and subsequently the formation of a TN-C-enriched ECM that contributes to fibroblast migration.

\section{Introduction}

FAK is a nonreceptor tyrosine kinase that localizes to focal adhesions, where it integrates and amplifies signals transduced to the cell interior by adhesion receptors and other extracellular effectors, including soluble growth factors (Schaller, 2001; Schwartz and Ginsberg, 2002). Comparison of FAK-wild-type and -null embryos has provided major clues regarding the functions of FAK in vivo (Furuta et al., 1995; Ilic et al., 1995, 1996). Mice lacking both copies of the fak gene die at E8.0-8.5 and exhibit a number of abnormalities, including severe morphogenetic defects, evident primarily in the axial mesodermal tissues and the cardiovascular system (Furuta et al., 1995). Analyses of cells isolated from FAK-null embryos suggest that FAK participates in embryogenesis not only by controlling cell proliferation and survival but also via its effects on cellular morphology and migration (Ilic et al., 1995, 1996; Owen et al., 1999). For example,

\footnotetext{
Address correspondence to Peter L. Jones, Dept. of Pediatrics, University of Colorado Health Sciences Center, 4200 East 9th Ave., B-131, Denver, CO 80262. Tel.: (303) 315-4497. Fax: (303) 315-1326.

E-mail: Peter.Jones@UCHSC.edu

Key words: ECM; homeobox genes; adhesion; FAK-null; transcription
}

cultured FAK-null embryonic fibroblasts possess a larger number of highly stable focal adhesions, and accordingly, display a circular morphology and a reduced capacity to migrate on fibronectin $(\mathrm{FN})^{*}$-coated surfaces. Stable expression of activated FAK in FAK-null cells, however, increases cell spreading and reestablishes migration on FN (Sieg et al., 1999). In terms of the mechanism whereby FAK controls cell migration, the most widely accepted paradigm is that activated FAK regulates the cycle of assembly and disassembly of focal adhesions, thereby allowing cells to dynamically interact with their underlying ECM (Ilic et al., 1997). Another possibility is that activated FAK controls the expression of ECM genes and proteins that contribute to a pro-migratory tissue microenvironment, yet this idea has not been fully explored.

Tenascin-C (TN-C) is an ECM glycoprotein expressed in developing tissues, as well as within remodeling adult tissues, such as wounds and tumors (Chiquet-Ehrismann et al., 1986; Jones and Jones, 2000). Numerous cellular functions

\footnotetext{
*Abbreviations used in this paper: EMSA, electrophoretic mobility shift assay; FN, fibronectin; FRNK, FAK-related nonkinase; HBS, homeodomain binding site; Prx1, paired-related homeobox 1; rPrx1, rat Prx1; TN-C, tenascin-C.
} 
Figure 1. TN-C expression correlates with FAK. (A) FAK-wild-type and -null fibroblasts were allowed to migrate toward $\mathrm{FN}$ for $3 \mathrm{~h}$ (left); ${ }^{*}, \mathrm{P}<$ 0.05. FAK-wild-type cells were allowed to migrate toward $\mathrm{FN}$ for $3 \mathrm{~h}$ in the presence of a control IgG or an anti-TN-C antibody (right); ${ }^{*}, \mathrm{P}<0.01$. (B) Semi-quantitative RT-PCR assays to assess the steady-state levels of TN-C and GAPDH mRNA levels in FAK-wild-type (+) and -null fibroblasts $(-)$. The number of PCR cycles used to amplify TN-C mRNA is indicated. (C) Western immunoblots for TN-C (top) and smooth muscle $\alpha$-actin (bottom) in FAK-wild-type $(+)$ and -null (-) fibroblasts. (D) Photomicrographs showing FAK-wild-type (+) and -null (-) fibroblasts plated onto FN for $24 \mathrm{~h}$ after immunostaining for TN-C, $\alpha$-actin, or vinculin (green). Cell nuclei were visualized with DAPI (blue). Bar, $30 \mu \mathrm{m}$.
A



B

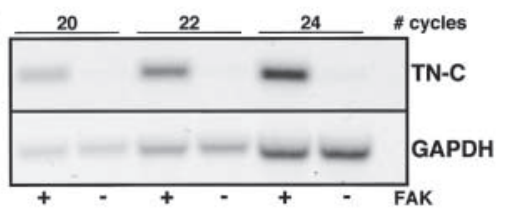

C

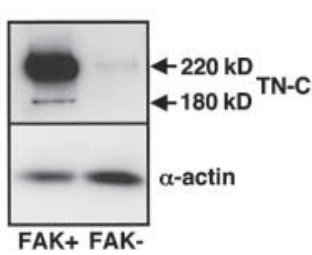

D

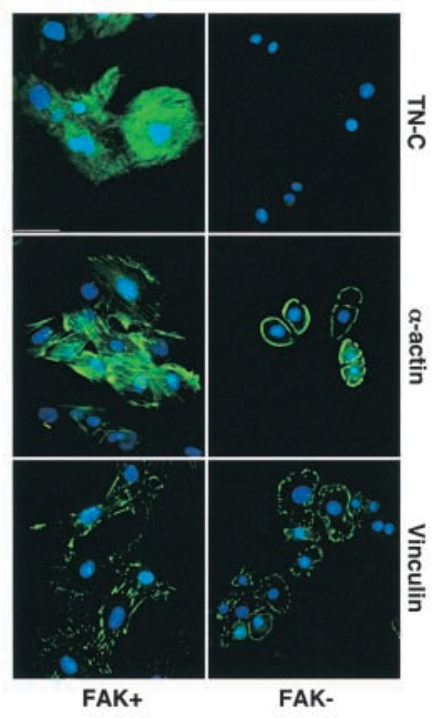

have been ascribed to TN-C, including the control of cellular proliferation, apoptosis, and differentiation (VrucinicFilipi and Chiquet-Ehrismann, 1993; Jones and Jones, 2000). Analyses of various cells and tissues have also shown that TN-C protein, (especially larger splice variants containing the TnfnA-D domain), is associated with a migratory phenotype in vivo and in tissue culture (Mackie et al., 1988; Halfter et al., 1989; Derr et al., 1997; Fischer et al., 1997). The idea that TN-C promotes cell migration is also supported by studies demonstrating that extracellular TN-C disassembles stable focal adhesions (Murphy-Ullrich et al., 1991; Chung et al., 1996). Furthermore, TN-C is able to reduce the strength of cell binding interactions with other ECM molecules, including FN (Lotz et al., 1989). Also, TN-C-null mice exhibit wound healing defects (Matsuda et al., 1999), and in vivo knockdown of TN-C expression in avian embryos attenuates neural crest cell migration (Tucker, 2001). Collectively, these and other studies indicate that TN-C represents an ECM constituent that is suitably poised to promote cell migration.

TN-C is induced by many of the same factors that activate FAK, including soluble growth factors, adhesion molecules, and biomechanical force (Chiquet-Ehrismann et al., 1995; Jones et al., 1999; Wang et al., 2001). For the most part, intracellular signals generated by these extracellular stimuli regulate TN-C expression at the transcriptional level (Chiquet-Ehrismann et al., 1995; Jones and Jones, 2000). Identifying transcription factors that control TN-C expression is therefore critical to understanding the regulation and tissuespecific functions of TN-C. Paired-related homeobox 1 (Prx1) and Prx2 encode transcription factors that induce TN-C gene transcription via their ability to interact with a homeodomain binding site (HBS) located within the proximal promoter region of the TN-C gene (Jones et al., 2001; Norris and Kern, 2001). Prx1 and Prx2 are not only expressed in the same locations as TN-C during embryogenesis and in remodeling adult tissues (Bergwerff et al., 1998; Jones et al., 2001) but they have also been shown to up-regulate TN-C gene transcription in response to changes in cell adhesion to the ECM (Jones et al., 2001). Although these latter studies indicate that an integrin-dependent signaling pathway might control TN-C gene transcription via its effects on Prx proteins, the upstream signaling molecules that mediate this response have not been identified. Given the central role that FAK plays in relaying integrin-dependent signals required for cell migration (Ilic et al., 1997), we hypothesized and showed that FAK controls TN-C-dependent cell migration via its ability to regulate the function of Prx1.

\section{Results}

\section{Activated FAK is required for expression of the pro-migratory ECM protein TN-C}

To determine whether FAK-dependent fibroblast migration toward FN relies on cellular interactions with TN-C, haptotactic migration assays were performed. Consistent with previous studies (Sieg et al., 1999), migration of FAK-wild-type cells through transwells undercoated with $\mathrm{FN}$ was significantly greater than that of FAK-null cells (Fig. $1 \mathrm{~A}$, left). To determine whether TN-C plays a role in this process, FAKwild-type fibroblasts were plated onto transwells either in the presence of an anti-TN-C antibody, or a control IgG. Although antibody treatment did not affect cell adhesion to the transwell surface (unpublished data), blocking cellular interactions with TN-C significantly reduced fibroblast migration toward FN (Fig. $1 \mathrm{~A}$, right). It should be noted that the TN-C antibody did not reduce the relative rate of cell migration to the levels observed with FAK-null cells, thereby indicating that FAK-dependent fibroblast migration relies on other molecules besides TN-C. Nevertheless, these experiments allowed us to hypothesize that FAK regulates fibroblast migration, at least in part, via its effects on TN-C expression.

RT-PCR using total RNA derived from FAK-wild-type and -null fibroblasts showed that TN-C mRNA expression was suppressed in the absence of FAK (Fig. 1 B). Western analysis and immunofluorescence studies also demonstrated that FAK-wild-type cells synthesized and deposited large amounts of extracellular TN-C protein, whereas FAK-null 
A

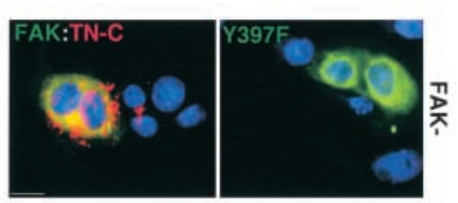

D



B
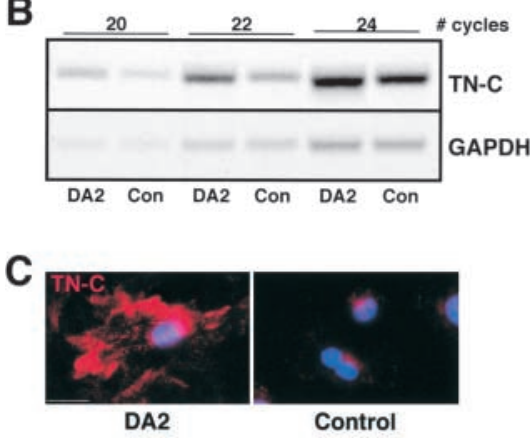

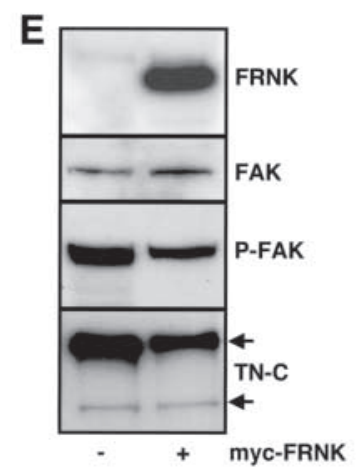

Figure 2. Activated FAK is required for TN-C expression. (A) Immunostaining for wild-type FAK fusion protein (left, green), or a kinase-dead FAK mutant $\left(\mathrm{Y}^{397} \mathrm{~F}\right.$; right, green), as well as $\mathrm{TN}-\mathrm{C}$ (red), in transiently transfected FAK-null (-) fibroblasts. Nuclei are stained blue with DAPI. Bar, $15 \mu \mathrm{m}$. (B) Semi-quantitative RT-PCR assays to assess the steady-state levels of TN-C and GAPDH mRNA levels in DA2 and control cells. The number of PCR cycles used to amplify TN-C mRNA is indicated. (C) Photomicrographs showing DA2 and control cells plated onto $\mathrm{FN}$ after immunostaining for TN-C (red). Cell nuclei were visualized with DAPI (blue). Bar, $30 \mu \mathrm{m}$. (D) Photomicrographs showing FAK-wild-type (+) cells replated onto FN after transient transfection with FRNK. Cells in the left panel were stained for the c-myc/FRNK fusion protein (red) and activated FAK ( $\mathrm{Y}^{397}$ phosphoFAK-specific antibody; green). Cells in the right panel were stained for the c-myc/FRNK fusion protein (red) and TN-C (green). Cell nuclei were visualized with DAPI (blue). Bar, $30 \mu \mathrm{m}$. (E) Western immunoblotting for FRNK, total FAK, phosphorylated FAK and TN-C in cells transiently transfected with a control vector (-), or with a plasmid encoding a c-myc/FRNK fusion protein (+).

cells expressed greatly reduced levels of TN-C (Fig. 1, C and D). Although two isoforms of TN-C were abundantly expressed in FAK-wild-type fibroblasts, the higher molecular mass $220-\mathrm{kD}$ isoform, which has been functionally linked to migrating cells, was by far the most evident (Fig. $1 \mathrm{C}$ ). Immunostaining for $\alpha$-actin and vinculin showed that TN-C expressing FAK-wild-type cells exhibit an elongated and polarized morphology, whereas TN-C-deficient FAK-null cells are circular and harbor a greater number of focal adhesions (Fig. 1 D). Thus, FAK not only correlates with cell migration and elongation, but also with TN-C expression.

Next, we determined whether FAK activity is required for TN-C expression. FAK-null fibroblasts were replated onto FN after transient transfection with plasmids encoding either an IL2R-FAK fusion protein (i.e., constitutively active FAK), or a mutated IL2R-FAK protein $\left(\mathrm{Y}^{397 \mathrm{~F}}\right)$, in which the $\mathrm{SH} 2$ binding site tyrosine has been substituted for phenylalanine (Tamura et al., 1999). Immunostaining for IL2R-FAK and TN-C in transfected cells showed that constitutive expression of wild-type FAK, but not the mutated form, increased extracellular TN-C deposition (Fig. 2 A). In parallel, we examined TN-C mRNA and protein expression using a FAK-null cell clone that was engineered to stably express FAK (i.e., DA2 cells; Sieg et al., 1999). The characteristics of DA2 cells have been described previously in detail (Sieg et al., 1999), including their restored ability to migrate toward FN. RT-PCR (Fig. 2 B) and immunofluorescence (Fig. 2 C) assays showed that TN-C expression is greater in DA2 fibroblasts than in controls. However, the basal levels of TN-C expression were greater in the control cells for DA2 when compared with FAK-null cells (Fig. $1 \mathrm{~B}$ ). A possible explanation for this difference may lie in the fact that DA2 and its control line represent hygromycin-selected clones. Because nonselected FAK-null cells are heterogeneous in nature, and they do express some TN-C, it is possible that clonal selection enriched for cells expressing higher basal levels of TN-C.

As a corollary to these experiments, FAK-wild-type cells were replated onto FN after transient transfection with a c-myc epitope-tagged plasmid encoding FAK-related nonkinase (FRNK), an endogenous and negative regulator of FAK activity (Schaller et al., 1993). As expected, immunofluorescence staining for the c-myc/FRNK fusion protein and activated FAK (using a $\mathrm{Y}^{397}$ phospho-FAK-specific antibody) showed that transient expression of FRNK, in $\sim 10 \%$ of the total cell population (unpublished data), suppressed FAK activity (Fig. 2 D, left, and Fig. 2 E). Moreover, inhibiting FAK activity via transient overexpression of FRNK also reduced TN-C expression (Fig. $2 \mathrm{D}$, right, and Fig. $2 \mathrm{E}$ ), yet this did not suppress the total levels of FAK protein (Fig. 2 E). These data establish that activated FAK is required for TN-C expression.

\section{Control of TN-C expression and fibroblast migration by Prx transcription factors}

Because FAK is required for TN-C expression and the paired-related homeobox genes Prx 1 and $P r \times 2$ regulate TN-C gene transcription via their ability to interact with an HBS within the TN-C gene promoter, we hypothesized that FAK-dependent migration toward FN might also rely on Prx transcription factors. To test this, FAK-wild-type cells were incubated with FITC-labeled, control, or antisense morpholino oligonucleotides, designed to specifically inhibit Prx 1 and Prx2 protein expression.

Phase-contrast and fluorescence microscopic imaging of FAK-wild-type cells demonstrated uptake of control and antisense oligonucleotides in more than $70 \%$ of treated cells (Fig. $3 \mathrm{~A}$ ), with no evidence of toxicity (unpublished data). Because morpholino oligonucleotides affect protein translation rather than mRNA stability, we next evaluated Prx1 and Prx 2 protein levels by Western immunoblotting. Antisense morpholino-treated fibroblasts expressed reduced levels of Prx 1 and Prx 2 protein, when compared with controls (Fig. 3 B). It should also be noted that an additional crossreactive band was detected in Western blots evaluating Prx1 expression, as reported previously (Chesterman et al., 2001). Targeted suppression of Prx1 and Prx 2 also resulted in 
Figure 3. Paired-related homeobox genes regulate TN-C and fibroblast migration. (A) Representative phase-contrast (top) and fluorescence (bottom) micrographs showing FAK-wild-type cells treated with FITC-labeled control or Prx1 and Prx2 antisense (AS) oligonucleotides. Bar, $30 \mu \mathrm{m}$. (B) Western immunoblotting for Prx1, Prx2, TN-C, and $\alpha$-actin in control and AS oligonucleotide-treated FAK-wild-type fibroblasts. The anti-Prx1 antibody recognizes two isoforms designated Prx1a and Prx1b. An additional cross-reactive band was also detected in both samples, as reported previously (Chesterman et al., 2001). (C) Haptotactic migration assays were used to evaluate fibroblast migration toward FN in control and AS-treated cells. Shown is relative cell migration between groups \pm SEM of triplicates from at least three independent experiments. ${ }^{*}, \mathrm{P}<0.05$.
A
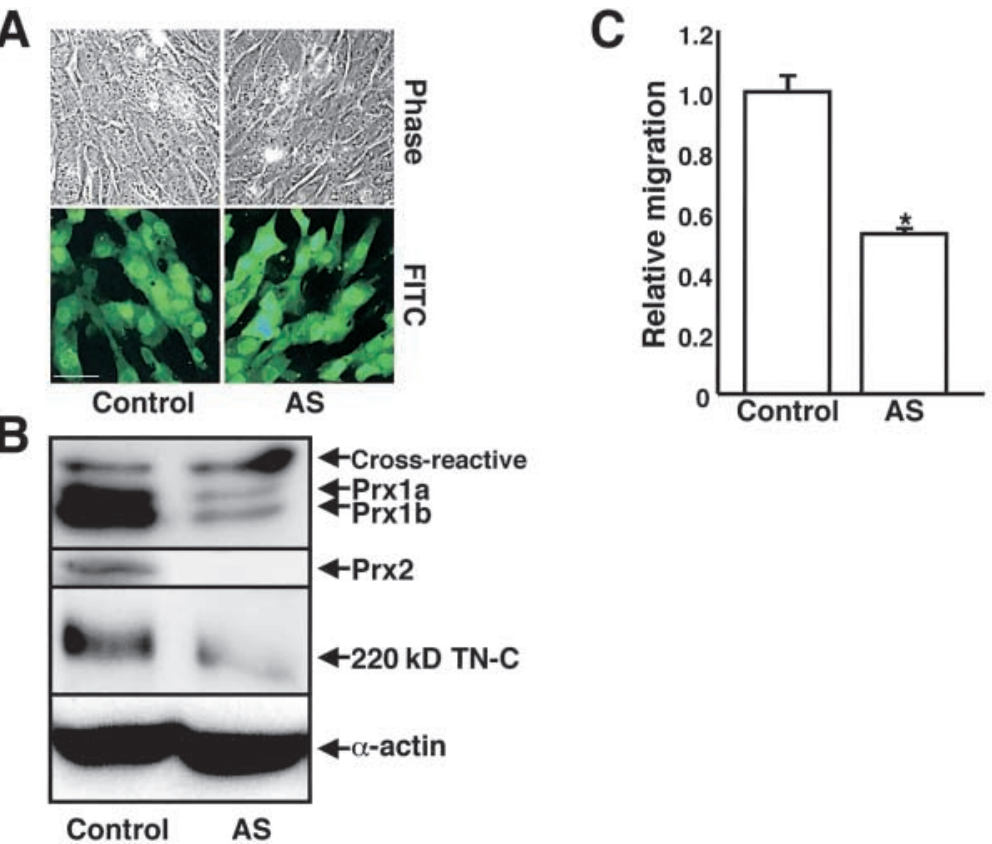

down-regulation of TN-C protein expression, whereas $\alpha$-actin protein expression remained unchanged (Fig. $3 \mathrm{~B}$ ). Having established a system in which expression of Prx 1 , $\operatorname{Prx} 2$, and TN-C are suppressed, we evaluated cell migration toward FN. Compared with control oligonucleotide-treated cells, haptotactic migration was significantly reduced in cultures treated with Prx antisense oligonucleotides (Fig. 3 C), despite the fact that cell adhesion to the transwell surface was unaffected (unpublished data). Thus, in addition to FAK and TN-C, Prx transcription factors are required for fibroblast migration toward FN.

\section{DNA-binding activity and induction of TN-C by Prx1}

FAK has been shown to promote the DNA-binding activity of the transcriptional effector STAT1, which in turn enhances fibroblast migration via its effect on gene transcription (Xie et al., 2001). To determine whether activated FAK promotes TN-C gene expression via its effect on Prx transcription factor binding to the TN-C gene promoter HBS, electrophoretic mobility shift assays (EMSAs) were performed. These experiments were also conducted in order to determine whether Prx1, Prx2, or a combination of both transcription factors, participate in FAK-dependent induction of TN-C gene expression.

FAK-wild-type fibroblast nuclear extracts incubated with a radiolabeled wild-type HBS probe formed three distinct DNA-protein complexes in EMSAs (Fig. 4 A, lane 2), whereas incubation of FAK-wild-type nuclear extracts with the mutated HBS probe failed to support complex formation (Fig. 4 A, lane 1). Similarly, DNA-protein complex formation did not occur when the wild-type or mutated HBS were incubated with nuclear extracts derived from FAK-null cells (Fig. 4 A, lanes 6 and 7). To determine whether Prx proteins were present in DNA-protein complexes, the wild-type probe and FAK-wild-type nuclear extracts were incubated with antibodies that recognize either Prx1 or Prx2. Treatment with the Prx1 antibody resulted in an almost complete disruption of the DNA-protein complexes (Fig. $4 \mathrm{~A}$, lane 3), whereas incubation with the antiPrx2 antibody, or a control IgG, had no effect (Fig. 4 A, lanes 4 and 5). These experiments demonstrate that Prx1 protein binding to the HBS fails to occur in the absence of FAK, and indicate that Prx 2 does not interact with the HBS in vitro, and is therefore unlikely to be a constituent of the FAK signaling pathway controlling TN-C expression in fibroblasts.

Having established that Prx1 interacts with the HBS in FAK-wild-type cells, we assessed whether exogenous Prx1 protein is able to bind to the HBS in the absence of FAK. Accordingly, in vitro transcribed/translated, recombinant rat Prx1 (rPrx1) protein was incubated with the wild-type HBS in the absence of nuclear extracts. As a control for protein binding specificity, recombinant RhoA ( $\mathrm{rRhoA}$ ) protein was incubated with the wild-type HBS probe. $r \operatorname{Prx} 1$ interacted with the HBS (Fig. $4 \mathrm{~A}$, lane 8), whereas rRhoA failed to bind (unpublished data). Moreover, inclusion of Prx1 antibody in these EMSAs confirmed that $\mathrm{rPrx} 1$ was present in the DNA-protein complexes (Fig. 4 A, lane 9). In contrast, a control IgG did not perturb complex formation (Fig. 4 A, lane 10). Although these data do not exclude the possibility that FAK modulates Prx1 binding to the HBS in vivo, they do indicate that Prx1 is able to bind to the HBS without FAK, and that FAK might control Prx 1 function in an alternative manner. Also, when compared with EMSAs performed using FAK-wild-type nuclear extracts, only two major complexes were detected in EMSAs involving rPrx1. Most likely, this is because mouse cells and tissues express two Prx1 isoforms, designated Prxla and Prx1b (Fig. 2 A; Chesterman et al., 2001). Furthermore, the appearance of two complexes using $\mathrm{rPRx} 1$ is likely due to premature translation termination, or initiation at a downstream methionine.

Because FAK is not essential for Prx1 protein binding to the HBS in vitro, we next wanted to determine whether 


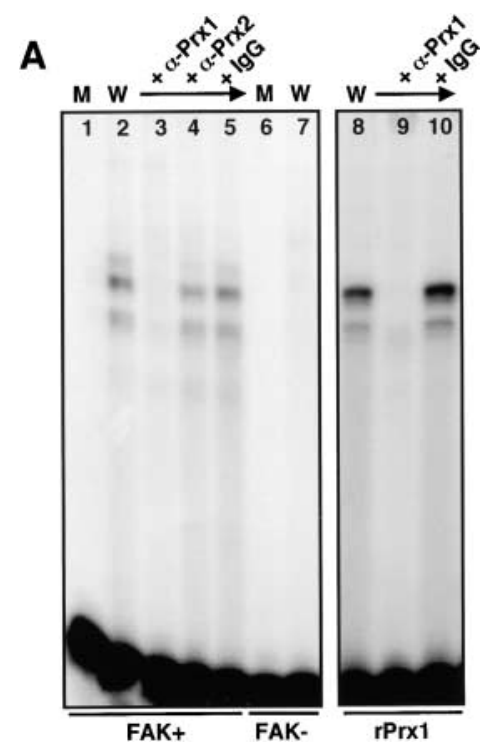

B

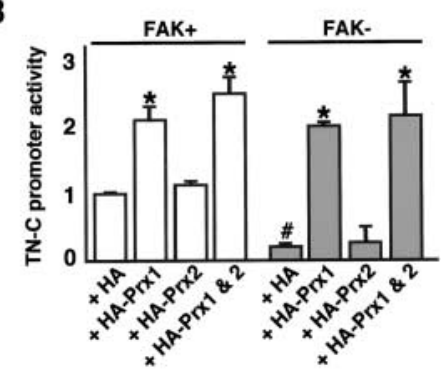

Figure 4. Prx1 interactions with the HBS and up-regulation of TN-C. (A) EMSAs using FAK-wild-type (+) and -null (-) fibroblast nuclear extracts, incubated with either a radiolabeled wild-type (W) or mutated (M) HBS probe. To determine the presence of Prx proteins in binding complexes, the wild-type probe and FAK-wild-type nuclear extracts were incubated with antibodies that recognize Prx1, Prx2, or a control IgG. In separate experiments, in vitro transcribed/translated, recombinant $r P r x 1$ protein was incubated with the wild-type-HBS in the absence of nuclear extracts. These complexes were also treated with an anti-Prx1 antisera or a control IgG. (B) TN-C promoter/luciferase gene reporter assays in FAKwild-type (+) or -null (-) cells cotransfected with a TN-C promoter/ luciferase gene reporter and $\mathrm{HA}$ epitope-tagged Prx expression vectors (i.e., HA-Prx1, HA-Prx2, or a combination of both). A $\beta$-galactosidase expression vector was included in each transfection to normalize for transfection efficiency. Shown is the relative fold increase in TN-C promoter activity \pm SEM of triplicates from at least three independent experiments. ${ }^{*}, \mathrm{P}<0.05$ between all groups. $\#, \mathrm{P}<0.05$ between $\mathrm{HA}$ vector alone transfected into FAK-wild versus -null cells.

Prx1 was able to transactivate the TN-C gene promoter in FAK-null cells. Accordingly, both FAK-wild-type or -null cells were cotransfected with a TN-C promoter/luciferase gene reporter plasmid that harbors the HBS, together with HA epitope-tagged Prx expression vectors, or an empty HA expression vector as a control. A $\beta$-galactosidase expression vector was also included to account for transfection efficiency. TN-C gene promoter activity was greater in FAKwild-type versus -null cells transfected with the control vector (Fig. 4 B), raising the possibility that FAK-null cells might express less endogenous Prx1 protein compared with
FAK-wild-type cells. In addition, Prx1 expression significantly increased TN-C gene promoter activity in both FAKwild-type and -null cells, when compared with the control vector (Fig. 4 B). On the other hand, transfection of FAKwild-type and -null cells with the Prx2 vector alone had no effect on basal levels of TN-C promoter activity observed in control-transfected cultures (Fig. 4 B). Similarly, cotransfection of Prx1 and Prx2 did not increase the levels of promoter activity recorded in cells transfected with Prx1 alone (Fig. 4 B). Therefore, consistent with the EMSA experiments, Prx1 is able to function independently of FAK, and it appears to be the predominant Prx transcription factor involved in the control of TN-C expression in fibroblasts.

\section{FAK is required for Prx1 expression}

To determine whether FAK regulates TN-C by inducing expression of Prx1, we conducted RT-PCR assays using total RNA isolated from FAK-wild-type and -null fibroblasts, which showed that the steady-state levels of Prx1 mRNA are suppressed in FAK-null cells (Fig. 5 A). Consistent with this, Western immunoblotting showed that expression of Prxla and Prx1b was suppressed in FAK-null cells (Fig. 5 B). Next, we directly determined whether FAK is required for Prx1 expression. It has been reported previously that although the Prx1 antibody recognizes a cross-reactive band in Western immunoblots, this antibody specifically recognizes Prx1 when used for immunocytochemistry (Chesterman et al., 2001). Immunostaining experiments confirmed that FAKnull cells do not express Prx1 protein (Fig. 5 C, left), whereas FAK-null cells transiently transfected with the plasmid encoding the IL2R-FAK wild-type fusion protein expressed Prx1 protein in their nuclei (Fig. 5 C, right). RTPCR and immunofluorescence experiments using the FAKreconstituted DA2 clone also showed that FAK is required for Prx $1 \mathrm{mRNA}$, and protein expression (Fig. 5, D and E). As with TN-C mRNA expression, basal levels of Prx1 expression were greater in control cells for DA2 (Fig. 5 A), when compared with FAK-null cells.

To ascertain whether activated FAK is required for Prx 1 expression, FAK-wild-type fibroblasts were transiently transfected with the myc-tagged FRNK expression vector. Immunostaining experiments showed that FAK-wild-type cells express nuclear Prx1 protein (Fig. 5 F, left), whereas overexpression of the myc-FRNK fusion protein reduced the appearance of Prx1 in the nucleus (Fig. 5 F, right). Together, these studies show that activated FAK is also required for Prx1 expression.

\section{Prx1 expression promotes TN-C-dependent migration in FAK-null fibroblasts}

The aforementioned studies indicate that FAK-dependent induction of Prx1 activates TN-C gene transcription, and subsequently the formation of a TN-C-enriched ECM that conceivably supports cell migration on FN. To determine whether Prx1 acts downstream from FAK in promoting TN-C-dependent migration toward $\mathrm{FN}$, we transiently transfected FAK-null fibroblasts with either a control HA vector or the HA-tagged Prx1 plasmid, together with a $\beta$-galactosidase expression construct. Transfection efficiency 
Figure 5. Prx1 expression depends on activated FAK. (A) Semi-quantitative RT-PCR assays to assess steady-state levels of Prx1 and GAPDH in FAKwild-type $(+)$ and -null ( - fibroblasts. The number of PCR cycles used to amplify Prx1 mRNA is indicated. (B) Western immunoblots for Prx1a and Prx $1 b$ (top) and $\alpha$-actin (bottom) in FAK-wild-type $(+)$ and - null $(-)$ fibroblasts. An additional crossreactive band was also detected in both samples. (C) Representative fluorescence micrographs assessing Prx1 immunoreactivity in FAK-null (-) fibroblasts (left), or in FAK-null fibroblasts transiently transfected with the IL2R-FAK wild-type fusion protein (right). FAK is shown in green, whereas Prx1 is shown in red. Nuclei are visualized by DAPI staining. Bar, $15 \mu \mathrm{m}$. (D) Semi-quantitative RT-PCR assays were used to assess the steady-state levels of Prx1 and GAPDH mRNA levels in DA2 cells (i.e., FAK-null cells stably transfected with FAK) and its control line (i.e., FAK-null cells stably transfected with pcDNA3.1). The number of PCR cycles used to amplify Prx 1 mRNA is indicated.

(E) Photomicrographs showing DA2 cells and its control line plated onto FN after immunostaining for Prx1 protein (red). Bar, $30 \mu \mathrm{m}$. (F) Photomicrographs showing FAK-wild-type (+) cells replated onto FN followed by immunostaining for Prx1 (left). FAK-wild-type cells were transiently transfected with the c-myc/FRNK fusion protein (right), and then stained for FRNK using c-myc antibody (green) and for Prx1 protein (red). Cell nuclei were visualized with DAPI (blue). Bar, $15 \mu \mathrm{m}$.

was the same in all cells (unpublished data). Thereafter, transfected cells were replated onto transwells undercoated with FN, either in the presence of a control IgG, or an antiTN-C antibody. Antibody treatment had no effect on cell adhesion to the transwell surface. In IgG-treated cultures, Prx1 expression in FAK-null cells promoted cell migration toward FN compared with control-transfected cells (Fig. 6 A), whereas the anti-TN-C antibody significantly inhibited this effect (Fig. $6 \mathrm{~A}$ ).

In keeping with a role for $\operatorname{Prx} 1$ in supporting fibroblast migration via the induction of TN-C, immunostaining experiments showed that FAK-null cells transfected with an
HA-Prx1 expression vector not only expressed TN-C protein, but that this led to the acquisition of a more elongated cell morphology on FN (Fig. 6 B). Also, chronic adhesion (i.e., $24 \mathrm{~h}$ ) of FAK-null fibroblasts to TN-C resulted in a loss of cortical actin around the cell perimeter, as well as the formation of numerous, small focal adhesions, identified through vinculin staining (Fig. $6 \mathrm{C}$ ). Together, these studies establish that Prx1 and TN-C promote fibroblast migration toward FN, and that both genes function downstream from FAK in regulating fibroblast migration.

Having shown that TN-C is necessary for Prx1-dependent FAK-null fibroblast migration toward FN, we next deter-

\section{Figure 6. Prx1 promotes TN-C-dependent} migration. (A) FAK-null fibroblasts transfected with an HA-control or HA-Prx1 expression vector were allowed to migrate for $3 \mathrm{~h}$ toward $\mathrm{FN}$ in a transwell migration assay, in the presence of control IgG or an anti-TN-C antibody. Transfection efficiency was the same for all cells. Shown is the relative cell migration from at least three independent experiments; ${ }^{*}, \mathrm{P}<0.05$. (B) Representative fluorescence micrograph showing Prx1 immunoreactivity in FAK-null fibroblasts transiently transfected with the HA-Prx1 expression vector. Cells were stained for Prx1 via HA (red) and TN-C (green). Nuclei are visualized by DAPI staining. Bar, $30 \mu \mathrm{m}$. (C) Immunofluorescence photomicrographs showing FAK-null (-) fibroblasts plated onto either FN or TN-C, and then 24 h later immunostained with antibodies recognizing $\alpha$-actin or vinculin (both in green). Cell nuclei were visualized with DAPI (blue). Bar, $20 \mu \mathrm{m}$. (D) Haptotactic migration assays were used to evaluate fibroblast migration toward TN-C or FN in FAKwild-type (+) and -null (-) fibroblasts. Shown is the relative cell migration from at least three independent experiments; ${ }^{*}, \mathrm{P}<0.05$.

\section{A}
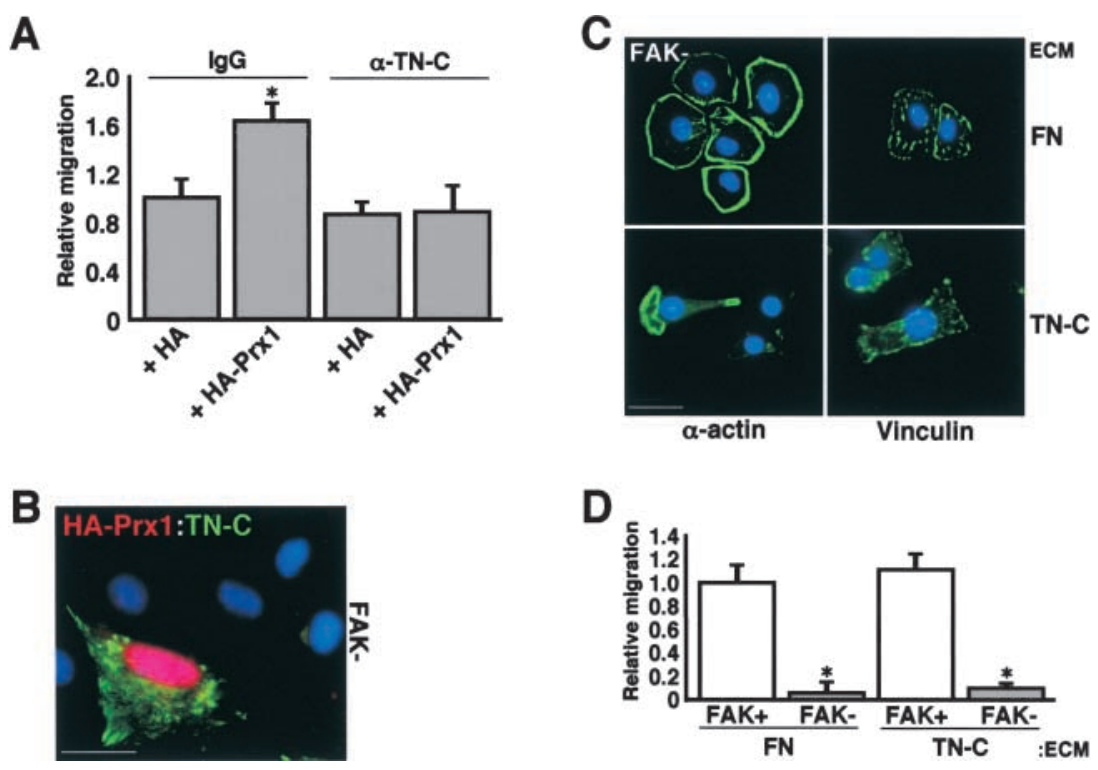
mined whether TN-C is able to rescue FAK-null cells from their migration defect. Accordingly, FAK-wild-type or -null cells were plated onto transwells undercoated with TN-C or FN. No differences in cell adhesion to the surface of the transwells were observed between groups (unpublished data). Identical to $\mathrm{FN}$, exogenous TN-C protein was unable to rescue the FAK-null cell migration defect (Fig. 6 D). Similarly, replating FAK-wild-type cells treated with Prx1 morpholino antisense oligonucleotides onto transwells undercoated with TN-C was also unable to restore FAK-null fibroblast migration (unpublished data). These experiments indicate that although TN-C is necessary for fibroblast migration, it is not sufficient. Therefore, FAK and Prx1 likely regulate expression of other genes required for cell migration.

\section{Prx1 and TN-C expression is suppressed in FAK-null embryos}

To determine whether our tissue culture studies were relevant in vivo, we evaluated Prx 1 and TN-C expression in FAK-wild-type and -null mouse embryos, harvested at E8.5-9.0. Prx1 and TN-C mRNA expression was reduced in FAK-null embryos compared with their age-matched, FAK-wild-type counterparts (Fig. 7 A). Parallel immunofluorescence studies of FAK-wild-type and -null embryos showed that TN-C protein expression is also reduced in FAK-null embryos (Fig. 7 B). Thus, expression of Prx1 and TN-C appears to depend on FAK during embryonic development.

Because development of FAK-null embryos is delayed when compared with their wild-type counterparts (Furuta et al., 1995), we reasoned that this might account for the lower levels of TN-C and Prx1 expression observed in FAK-null embryos. However, FN-null embryos, which are also arrested in development and exhibit a phenotype that overlaps with FAK-null embryos (George et al., 1993), did not show reduced levels of Prx1 and TN-C mRNA, when compared with their wild-type counterparts (unpublished data). These data indicate that Prx1 and TN-C expression levels are reduced as a direct result of loss of FAK in vivo.
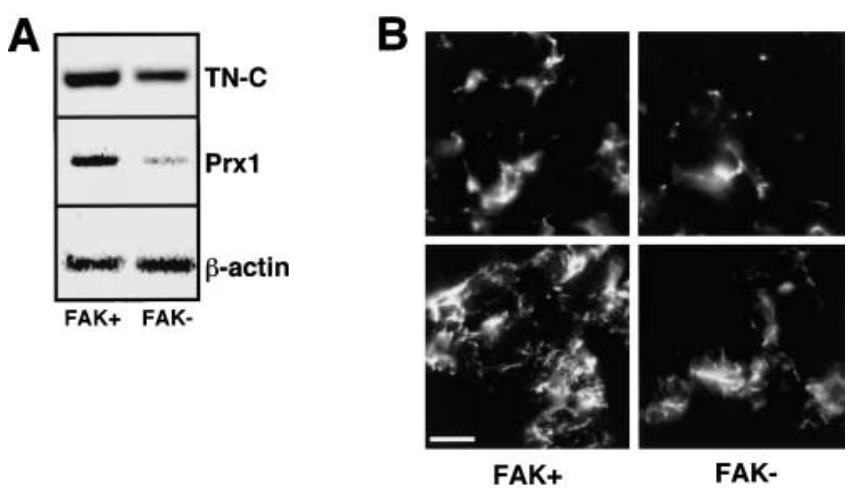

Figure 7. Expression of TN-C and Prx1 in FAK-null embryos. (A) Semi-quantitative RT-PCR assays to compare the steady-state levels of TN-C, Prx1, and $\beta$-actin mRNAs in FAK-wild-type $(+)$ and -null (-) E8.5 embryos. (B) Representative fluorescence micrographs showing immunostaining of headfold region of FAK-wild-type (+) and -null (-) E8.5 embryos for TN-C (green). Two different sections are shown. Bar, $20 \mu \mathrm{m}$.

\section{Discussion}

Many of the intracellular events that regulate fibroblast migration in response to activation of FAK have been defined, yet little is known about the transcription factors and target genes that lie downstream from FAK, and how these might control cell migration. Here, we showed that FAK is essential for the expression of Prx1, a transcription factor that activates the TN-C gene promoter. Moreover, we have demonstrated that Prx1-dependent induction of TN-C plays a role in fibroblast migration toward FN.

Although the exact mechanism whereby FAK induces expression of Prx1 has not yet been elucidated, it is known that alterations in cell adhesive interactions activate FAK (Ilic et al., 1997), and induce expression of Prx1 and TN-C (Jones et al., 1997, 2001). For example, when vascular smooth muscle cells are cultured on polymeric type I collagen, phosphorylation of FAK (unpublished data) and expression of Prx 1 and TN-C is suppressed (Jones et al., 2001), whereas monomeric type I collagen has the opposite effect. Because monomeric collagen is predominantly a ligand for $\alpha_{v} \beta_{3}$ integrins (Jones et al., 1997), and blockade of this receptor suppresses TN-C gene transcription (Jones et al., 1999), it is plausible that $\alpha_{v} \beta_{3}$ integrin-dependent activation of FAK promotes Prx1 expression. A number of FAK-dependent intracellular mediators, including c-src, PI3K, JNK, and ERK MAPKs, might induce Prx1 gene expression. However, pharmacological blockade of these signaling effectors in FAKwild-type cells appears to have no effect on the steady-state levels of Prx 1 mRNA expression (unpublished data). Ongoing studies will define alternative integrin-activated signaling mediators that regulate Prx1 gene expression via FAK.

In addition to changes in cell adhesion, alterations in cell and tissue biomechanics regulate TN-C expression (Chiquet-Ehrismann et al., 1994; Jones et al., 1997, 2002). Given that biological responses to externally applied mechanical force appear to be sensed and transduced to the cell interior by FAK (Wang et al., 2001), it is possible that biomechanical activation of FAK controls TN-C through the induction of Prx1. In addition to identifying the extrinsic and intracellular factors that control FAK-dependent induction of Prx 1 , it will be necessary to identify the nature of the transacting factors and cis elements within the Prx1 gene promoter that might respond to FAK. As stated earlier, Xie and colleagues (2001) demonstrated that integrins and FAK control the DNA-binding activity of the transcriptional effector Stat1. Moreover, FAK-dependent activation of Stat1 resulted in stimulation of cell migration. Because the downstream genes regulated in response to FAK-dependent activation of Stat1 have not yet been identified, it will be of interest to determine whether Stat 1 regulates TN-C by controlling Prx1 expression.

Focal adhesion formation is not only controlled by FAK but also by the small GTPase Rho (Ridley and Hall, 1992). Both activation and inactivation of Rho diminishes cell migration (Takaishi et al., 1993; Ren et al., 2000), suggesting that FAK controls focal adhesion turnover by modulating Rho activity. Consistent with this, FAK-wild-type fibroblasts transiently reduce Rho activity after initial adhesion and spreading to FN, whereas FAK-null cells fail to suppress 
Rho activity (Ren et al., 2000). Because TN-C suppresses Rho activation (Wenk et al., 2000), a tenable working hypothesis is that FAK-dependent induction of TN-C suppresses Rho activity in order to facilitate cell spreading and migration.

Although we showed that exogenous TN-C is unable to rescue the FAK-null fibroblast migration defect, we were able to demonstrate that endogenously produced TN-C plays a role in migration of FAK-wild-type cells toward FN, as well as for migration of FAK-null cells expressing Prx1. These data indicate that FAK and Prx1 regulate other promigratory genes besides TN-C. The notion that homeobox genes simultaneously regulate more than one target gene involved in regulating cell adhesion events is supported by studies demonstrating that during the invasive stage of angiogenesis, HoxD3 is required for expression of the proangiogenic molecules, $\beta_{3}$ integrins, and urokinase plasminogen activator (Boudreau et al., 1997).

Our studies possibly indicate that exogenous TN-C destabilizes focal adhesions in FAK-null fibroblasts. Clearly, this latter response would not be due to an autocrine loop involving TN-C-dependent activation of FAK. However, in the absence of FAK, a FAK-related protein tyrosine kinase, designated Pyk2 is upregulated (Avraham et al., 2000). Perhaps TN-C controls focal adhesion turnover in FAK-null cells using Pyk2. In terms of the TN-C cell surface receptors involved in remodeling focal adhesions, TN-C represents a ligand for multiple integrins (Jones and Jones, 2000). It has also been shown that TN-C interacts with cell surface annexin II via the TnfnA-D domain, and that this interaction perturbs focal adhesion integrity (Chung et al., 1996). Studies are now under way to identify which TN-C receptors control focal adhesion turnover in both FAK-wild-type and -null fibroblasts.

Finally, our tissue culture findings appear to be relevant to the function of FAK in vivo, given that expression of Prx 1 and TN-C is reduced in FAK-null embryos. However, Prx 1 and TN-C expression levels appear to be relatively higher in FAK-null embryos versus their cultured counterparts. Therefore, it is possible that nonfibroblast cell types within the embryo do not rely on FAK to regulate Prx 1 and TN-C. Alternatively, functional compensation by FAK-related molecules might account for the differences observed in vivo and in tissue culture. Microenvironmental factors and cell-cell interactions that are lost in tissue culture might also contribute to differences in Prx 1 and TN-C expression in vivo and in culture. It is also important to note that although TN-C-null mice exhibit wound repair defects that likely reflect cell migration deficits (Matsuda et al., 1999), these animals do not phenocopy either FAK-null or Prx1-null mice, both of which display vascular anomalies (Saga et al., 1992; Furuta et al., 1995; Bergwerff et al., 2000). FAK and/or Prx1-dependent induction of other pro-migratory ECM proteins that compensate for TN-C might account for these differences. Future avenues of investigation examining how FAK controls signal transduction pathways and gene networks involving Prx1 and its downstream targets will no doubt shed light onto how these molecules modulate cell migration in embryonic development and disease.

\section{Materials and methods}

\section{Reagents}

$\mathrm{TN}-\mathrm{C}$ and FN protein were purchased from CHEMICON International, Inc. Type I Collagen was purchased from Cohesion Labs. DME was purchased from Cellgro. Lipofectamine Plus and Superscript II RT were purchased from GIBCO BRL. FBS was purchased from Gemini Bio-Products. ReadyTo-Go RT-PCR beads, ECL+ kit, and Vistra Green were purchased from Amersham Biosciences. Normal goat serum was purchased from SigmaAldrich. PFA was purchased from EMS. Effectene transfection reagent and Rneasy Mini kits were purchased from QIAGEN. Hematoxylin and Vectashield mounting medium were purchased from Vector Laboratories.

Rabbit polyclonal $\alpha$-Prx 1 and $\alpha$-Prx 2 antisera have been described previously (Chesterman et al., 2001). Mouse mAbs smooth muscle $\alpha$-actin (1A4), $\alpha$-c-myc (9E10), $\alpha$-vinculin (VIN-11-5), and species-specific HRPconjugated secondary antibodies were obtained from Sigma-Aldrich. Rabbit polyclonal $\alpha$-FAK $(\mathrm{C}-20)$ and normal rabbit IgG were obtained from Santa Cruz Biotechnology, Inc. Rabbit polyclonal $\alpha-F A K\left(\mathrm{pY}^{397}\right)$ was obtained from Biosource International. Rat monoclonal $\alpha$-HA (3F10) was obtained from Roche. Mouse monoclonal $\alpha-$ IL-2 receptor (7G7/B6) was obtained from Upstate Biotechnology. Rabbit polyclonal $\alpha$-chicken TN-C antibody was obtained from CHEMICON International, Inc. Goat $\alpha$-rabbit (Alexa 488- and rhodamine-conjugated) and goat $\alpha$-mouse (Alexa 488and rhodamine-conjugated) were obtained from Molecular Probes. Goat $\alpha$-rat (Cy 3-conjugated) was obtained from Jackson ImmunoResearch Laboratories. Goat $\alpha$-rat (Texas red-conjugated) was obtained from Vector Laboratories.

CMV-promoter-driven dominant negative IL2R-FAK ${ }^{\mathrm{Y} 397 \mathrm{~F}}$ and constitutively active IL2R-FAK were a gift from Dr. K.M. Yamada (National Institutes of Health [NIH], Bethesda, MD) to Dr. M. Weiser-Evans. pCMVmycFRNK was a gift from Dr. J.T. Parsons (University of Virginia, Charlottesville, VA) to Dr. M. Weiser-Evans. TN7, a TN-C promoter construct ligated into a promoter-less PA3-Luc vector has been described previously (Jones et al., 2001). pCMV-HA was obtained from CLONTECH Laboratories, Inc. Rhox-14 (pCMV-HA) and Prx2-14 (pCMV-HA) were generated by EcoRI digestion of myc-tagged Prx1 and Prx2 expression vectors (Jones et al., 2001), subcloning into pBluescript SK (Stratagene), followed by Kpnl and partial Notl digestion, and then ligation into Notl-Kpnl digested pCMV-HA.

\section{Cell culture and transfection assays}

All cell lines were routinely maintained in complete DME supplemented with $4 \mathrm{mM}$ L-glutamine, $100 \mu \mathrm{M}$ NEAAs, $100 \mathrm{U} / \mathrm{ml}$ P/S plus $10 \%$ FBS. FAK-wild-type and -null fibroblasts were derived from E8.5 mice (Ilic et al., 1995). Both cell lines carry mutations in the p53 gene introduced by crossing heterozygous mice for FAK and p53 alleles (Furuta et al., 1995). DA2 represents a clone of FAK-null cells that were stably transfected with pcDNA3.1 HA-tagged FAK, whereas the control line represents FAK-null cells stably transfected with pcDNA3.1 empty vector (Sieg et al., 1999).

Plasmids IL2R-FAK ${ }^{\mathrm{Y} 97 \mathrm{~F}}$, IL2R-FAK, pCMV-mycFRNK, or pCMV-myc were transfected into either FAK-wild-type or -null fibroblasts using Effectene transfection reagent, whereas Rhox-14 (i.e., Prx1 cDNA), Prx2-14, pCMV-HA, and pCMV- $\beta$-galactosidase were transfected into FAK-null fibroblasts using Lipofectamine plus. For luciferase assays, FAK-wild-type and -null fibroblasts were transfected via electroporation (geneZAPPER; IBI) with $2 \mu \mathrm{g}$ TN7 plasmid, $2 \mu \mathrm{g}$ pCMV- $\beta$-galactosidase, and either $4 \mu \mathrm{g}$ pCMV-HA, or $2 \mu \mathrm{g}$ of both Rhox-14 and pCMV-HA, or $2 \mu \mathrm{g}$ of both Prx214 and pCMV-HA, or $2 \mu \mathrm{g}$ of both Rhox-14 and Prx2-14. Cells were transfected in complete DME plus 10\% FBS, before being replated. Media were changed to complete DME plus $2 \%$ FBS for $48 \mathrm{~h}$, and then extracts were prepared in reporter lysis buffer containing protease inhibitors. Luciferase activity and/or $\beta$-galactosidase activity were determined to evaluate TN-C promoter activity or transfection efficiency, respectively (Jones et al., 2001).

\section{Antisense morpholino oligonucleotides}

FITC-labeled antisense Prx1 (5'-GAACGTGCCCGTAGCTGGAGGTCAT$\left.3^{\prime}\right)$, antisense Prx2 (5'-GGCCGCGCTGTCCATGCCCTGGC-3'), and control (5'-CCTCTTACCTCAGTTACAATTTATA-3') morpholinos were purchased from Gene Tools LLC. $3.6 \mathrm{nM}$ morpholinos were delivered to cells cultured in 35-mm-diam tissue culture dishes in the presence of $1.45 \mu \mathrm{M}$ EPEI special delivery solution (Gene Tools LLC) in complete DME. After incubating at $37^{\circ} \mathrm{C}$ for $3 \mathrm{~h}$, media were changed to complete DME plus $10 \%$ FBS for $24 \mathrm{~h}$. Thereafter, cells were serum-starved before using in subsequent assays. Uptake of FITC-labeled oligonucleotides was assessed using an (Axiovert; Carl Zeiss Microlmaging, Inc.) inverted fluorescence microscope with appropriate FITC filters. 


\section{Western immunoblotting}

Protein extracts were prepared in $1 \times$ RIPA buffer or $1 \times$ cell lysis buffer (Cell Signaling) and total protein quantified using a Bio-Rad assay. Samples were separated on $8-16 \%$ polyacrylamide gels, transferred to polyvinyldifluoride membranes, which were blocked in wash buffer $(10 \mathrm{mM}$ Tris, $\mathrm{pH}$ $8,150 \mathrm{mM} \mathrm{NaCl}$, and $0.1 \%$ Tween 20) plus $5 \%$ milk. Blots were incubated with primary antibodies $(1.16 \mu \mathrm{g} / \mathrm{ml} \alpha-\mathrm{TN}-\mathrm{C}, 0.81 \mu \mathrm{g} / \mathrm{ml} \alpha$-Prx 1 , $0.252 \mu \mathrm{g} / \mathrm{ml} \alpha-\operatorname{Prx} 2,2.93 \mu \mathrm{g} / \mathrm{ml} \alpha-\alpha$ smooth muscle actin, $1 \mu \mathrm{g} / \mathrm{ml} \alpha-F A K$, $0.35 \mu \mathrm{g} / \mathrm{ml} \alpha-\mathrm{pY}^{397}$-FAK, and 1:100 $\alpha-\mathrm{c}-\mathrm{myc}$ [for FRNK]) in wash buffer plus $5 \%$ milk, rinsed in wash buffer, and incubated with HRP-conjugated secondary antibody $(1: 5,000)$. Proteins were detected using an ECL+ kit.

\section{Immunostaining}

Cells were fixed with $4 \%$ PFA in PBS. Embryos were fixed in $4 \%$ PFA, and then embedded and frozen in OCT media. 5- $\mu \mathrm{m}$ frozen embryonic sections were fixed after in $4 \%$ PFA before immunostaining. All cells and tissues were rinsed in PBS, and permeabilized in PBS containing $0.1 \%$ Triton $\mathrm{X}-100$, before being blocked in 10\% normal goat serum in PBSA (PBS plus $0.1 \%$ BSA), and incubated with primary antibodies in wash buffer. After addition of primary antibodies $(1.16 \mu \mathrm{g} / \mathrm{ml} \alpha-\mathrm{TN}-\mathrm{C}, 22 \mu \mathrm{g} / \mathrm{ml} \alpha-\alpha$ smooth muscle actin, $30 \mu \mathrm{g} / \mathrm{ml} \alpha$-vinculin, 1:100 $\alpha$-c-myc, $0.81 \mu \mathrm{g} / \mathrm{ml} \alpha-\operatorname{Prx} 1,2.5$ $\mu \mathrm{g} / \mathrm{ml} \alpha-\mathrm{HA}, 16.7 \mu \mathrm{g} / \mathrm{ml} \alpha-\mathrm{IL} 2 \mathrm{R}, 2 \mu \mathrm{g} / \mathrm{ml} \alpha-\mathrm{FAK}$, and $3.5 \mu \mathrm{g} / \mathrm{ml} \alpha-\mathrm{pY}^{397}$ FAK), samples were rinsed with PBSA, followed by incubation with species-specific fluorescein- or Texas red-conjugated antibodies (Molecular Probes, Inc.), diluted 1:100 in wash buffer. Slides were rinsed in PBS, before being mounted in Vectashield medium containing DAPI. Specimens were visualized using an inverted fluorescence microscope.

\section{Semi-quantitative RT-PCR}

First strand cDNA was synthesized from total RNA using Superscript II. A typical $25-\mu$ I PCR reaction contained one RT-PCR bead, $1 \mu \mathrm{l}$ CDNA, and $0.33 \mu \mathrm{M}$ of each of the following primers: Prx1, 5'-ACCTCCAGCTACGGGCACGTT-3' and 5'-ATGGCTCGCTCATTCCTGCGGAA-3' (for 23, 25, or 27 cycles); TN-C, 5'-GGAGTTGCTGGTATCGTCTCTAAGG-3' and 5'-ACACCTGCCATCCAAACACATC-3' (for 20, 22, or 24 cycles); $\beta$-actin, $5^{\prime}$-CATCCGTAAAGACCTCTATGCCAAC-3' and 5'-CAAAGAAAGGGTGTAAAACGCAGC-3' (for 15 cycles); and GAPDH, 5'-TGGGGCCAAAAGGGTCATCATCTC-3' and 5'-GCCGCCTGCTTCACCACCTTCTT$3^{\prime}$ (for 18,20 , or 22 cycles). PCR reaction conditions were as follows: $94^{\circ} \mathrm{C}$ for $1 \mathrm{~min}, 59.2^{\circ} \mathrm{C}(\mathrm{GAPDH})$ or $58.1^{\circ} \mathrm{C}$ (all others) for $1 \mathrm{~min}$, and then $72^{\circ} \mathrm{C}$ for $1 \mathrm{~min}$. Products were separated on $1-1.4 \%$ agarose $/ 1 \times$ TBE gels containing Vistra Green $(1: 10,000)$, and were visualized using a Storm 860 scanner, and quantified with Imagequant software.

\section{Electrophoretic mobility shift assays}

Nuclear extracts were prepared using a nuclear extract kit (Activemotif). A wild-type probe (5'-CGAGTTAATTAACCTGC-3' and 5'-GCAGGTTAATTAACTCG-3') containing the Prx HBS (underlined), and mutant probe (5'CGAGTTAACTCACCTGC-3' and 5'-GCAGGTGAGTTAACTCG-3') were annealed, gel purified, then $\left.\gamma={ }^{32} \mathrm{P}\right] A T P$ (PerkinElmer) end-labeled by polynucleotide kinase (Roche). $6 \mu \mathrm{g}$ nuclear extract was mixed with $25,000 \mathrm{cpm}$ probe in $4 \%$ Ficoll, $100 \mathrm{mM} \mathrm{KCl}, 12 \mathrm{mM}$ HEPES, $0.05 \% \mathrm{NP}-$ $40,50 \mu \mathrm{g} / \mathrm{ml} \mathrm{BSA}, 37.5 \mu \mathrm{g} / \mathrm{ml} \mathrm{dldC}$ at $37^{\circ} \mathrm{C}$ for $1 \mathrm{~h}$. Where indicated, $2 \mu \mathrm{l}$ of recombinant Prx1 protein (generated with TNT-coupled Wheat Germ Extract System; Promega) was added before incubation. Where indicated, antibodies were added (100 ng $\alpha$-Prx1, $\alpha$-Prx2, or normal rabbit IgG), 30 min after the reaction was initiated. Samples were loaded directly onto a $10 \%$ native polyacrylamide gel and electrophoresed at $45 \mathrm{~mA}$ for $2 \mathrm{~h}$. Gels were exposed and scanned using a Storm 860 scanner.

\section{Haptotactic migration assays}

Transwell chambers (8- $\mu \mathrm{m}$-diam pore size; Corning) were prepared by precoating the under surface of the polycarbonate membrane with $10 \mu \mathrm{g} /$ $\mathrm{ml} \mathrm{FN}$ protein in PBS for $2 \mathrm{~h}$ at $37^{\circ} \mathrm{C}$. FAK-wild-type and -null cells were serum-starved (complete DME plus $0.5 \%$ FBS) for $48 \mathrm{~h}$, before being plated onto transwells (Sieg et al., 1999). Transiently transfected and morpholino-treated cells were maintained in complete DME plus 10\% FBS (no $\mathrm{P} / \mathrm{S}$ ) for $36 \mathrm{~h}$ after transfection, before 48 -h serum starvation. 30,000 cells (in complete DME plus $0.5 \%$ BSA) were added to PBS-washed upper chambers of the transwell and were incubated for $3 \mathrm{~h}$ at $37^{\circ} \mathrm{C}$. Where indicated, normal rabbit IgG (DakoCytomation) or $\alpha-\mathrm{TN}-\mathrm{C}$ Ab (both at $20 \mu \mathrm{g} /$ $\mathrm{ml}$ ) were included in the culture media. Cells were fixed in 4\% PFA, and then stained with hemotoxylin. Cells on the upper surface of the membrane were removed with a cotton tip applicator. Cells on the lower surface were counted using phase microscopy. A minimum of three randomly chosen fields was counted per transwell, and a minimum of three tran- swells was used for each experimental group. All experiments were performed in triplicate.

\section{FAK-wild-type and -null embryos}

Heterozygous lines of FAK and FN mice were housed and bred in an environmentally controlled room at the UCSF according to institutional guidelines. Genotypes were determined by PCR analysis (Furuta et al., 1995). Embryos were isolated at E8.5. Placental cone and yolk sac were used for genotyping, and the embryos were either snap frozen and kept at $-80^{\circ} \mathrm{C}$ for RT-PCR analyses, or fixed in $3.8 \%$ PFA/PBS overnight at $4^{\circ} \mathrm{C}$, for immunostaining. Fixed embryos were washed three times in PBS, infiltrated with $5-15 \%$ sucrose followed by OCT compound (Miles Scientific), and frozen in liquid nitrogen. $5-\mu \mathrm{m}$ thick sections were prepared using a cryostat (Slee International, Inc.)

\section{Statistics}

All statistical assessments were compared by one-way analysis of variance (ANOVA) and ANOVA post-hoc analysis using Statview. P < 0.05 was considered statistically significant. Values were presented as means \pm SEM.

We would like to thank P. Garl (University of Colorado Health Sciences Center) for her excellent technical assistance, and Dr. N. Boudreau (University of California, San Francisco) for her helpful advice. Dr. D. Schlaepfer (The Scripps Research Institute, La Jolla, CA) provided DA2 cells and its companion line, and gave us many useful comments.

This project was supported by NIH grants (1 R01 HL68798-01 and 2 P50 HL57144-06) to P.L. Jones.

Submitted: 21 February 2003

Revised: 21 February 2003

Accepted: 7 March 2003

\section{References}

Avraham, H., S.Y. Park, K. Schinkmann, and S. Avraham. 2000. RAFTK/Pyk2mediated cellular signalling. Cell. Signal. 12:123-133.

Bergwerff, M., A.C. Gittenberger-de Groot, M.C. DeRuiter, L. van Iperen, F. Meijlink, and R.E. Poelmann. 1998. Patterns of paired-related homeobox genes PRX1 and PRX2 suggest involvement in matrix modulation in the developing chick vascular system. Dev. Dyn. 213:59-70.

Bergwerff, M., A.C. Gittenberger-de Groot, L.J. Wisse, M.C. DeRuiter, A. Wessels, J.F. Martin, E.N. Olson, and M.J. Kern. 2000. Loss of function of the $\operatorname{Prx} 1$ and Prx2 homeobox genes alters architecture of the great elastic arteries and ductus arteriosus. Virchows Arch. 436:12-19.

Boudreau, N., C. Andrews, A. Srebrow, A. Ravanpay, and D.A. Cheresh. 1997. Induction of the angiogenic phenotype by Hox D3. J. Cell Biol. 139:257-264.

Chesterman, E.S., G.D. Gainey, A.C. Varn, R.E. Peterson, Jr., and M.J. Kern. 2001. Investigation of $\operatorname{Prx} 1$ protein expression provides evidence for conservation of cardiac-specific posttranscriptional regulation in vertebrates. Dev. Dyn. 222:459-470.

Chiquet-Ehrismann, R., E.J. Mackie, C.A. Pearson, and T. Sakakura. 1986. Tenascin: an extracellular matrix protein involved in tissue interactions during fetal development and oncogenesis. Cell. 47:131-139.

Chiquet-Ehrismann, R., M. Tannheimer, M. Koch, A. Brunner, J. Spring, D. Martin, S. Baumgartner, and M. Chiquet. 1994. Tenascin-C expression by fibroblasts is elevated in stressed collagen gels. J. Cell Biol. 127:2093-2101.

Chiquet-Ehrismann, R., C. Hagios, and S. Schenk. 1995. The complexity in regulating the expression of tenascins. Bioessays. 17:873-878.

Chung, C.Y., J.E. Murphy-Ullrich, and H.P. Erickson. 1996. Mitogenesis, cell migration, and loss of focal adhesions induced by tenascin- $\mathrm{C}$ interacting with its cell surface receptor, annexin II. Mol. Biol. Cell. 7:883-892.

Derr, L.B., R. Chiquet-Ehrismann, R. Gandour-Edwards, J. Spence, R.P. Tucker. 1997. The expression of tenascin-C with the AD1 variable repeat in embryonic tissues, cell lines and tumors in various vertebrate species. Differentiation. 62:71-82.

Fischer, D., R.P. Tucker, R. Chiquet-Ehrismann, and J.C. Adams. 1997. Celladhesive responses to tenascin- $\mathrm{C}$ splice variants involve formation of fascin microspikes. Mol. Biol. Cell. 8:2055-2075.

Furuta, Y., D. Ilic, S. Kanazawa, N. Takeda, T. Yamamoto, and S. Aizawa. 1995. Mesodermal defect in late phase of gastrulation by a targeted mutation of focal adhesion kinase, FAK. Oncogene. 11:1989-1995.

George, E.L., E.N. Georges-Labouesse, R.S. Patel-King, H. Rayburn, and R.O. 
Hynes. 1993. Defects in mesoderm, neural tube and vascular development in mouse embryos lacking fibronectin. Development. 119:1079-1091.

Halfter, W., R. Chiquet-Ehrismann, and R.P. Tucker. 1989. The effect of tenascin and embryonic basal lamina on the behavior and morphology of neural crest cells in vitro. Dev. Biol. 132:14-25.

Ilic, D., Y. Furuta, S. Kanazawa, N. Takeda, K. Sobue, N. Nakatsuji, S. Nomura, J. Fujimoto, M. Okada, T. Yamamoto, and S. Aizawa. 1995. Reduced cell motility and enhanced focal adhesion contact formation in cells from FAKdeficient mice. Nature. 377:539-544.

Ilic, D., S. Kanazawa, Y. Furuta, T. Yamamoto, and S. Aizawa. 1996. Impairment of mobility in endodermal cells by FAK deficiency. Exp. Cell Res. 222:298303.

Ilic, D., C.H. Damsky, and T. Yamamoto. 1997. Focal adhesion kinase: at the crossroads of signal transduction. J. Cell Sci. 110:401-407.

Jones, F.S., and P.L. Jones. 2000. The tenascin family of ECM glycoproteins: structure, function, and regulation during embryonic development and tissue remodeling. Dev. Dyn. 218:235-259.

Jones, F.S., R. Meech, D.B. Edelman, R.J. Oakey, and P.L. Jones. 2001. Prx1 controls vascular smooth muscle cell proliferation and tenascin- $\mathrm{C}$ expression and is upregulated with Prx2 in pulmonary vascular disease. Circ. Res. 89: 131-138.

Jones, P.L., J. Crack, and M. Rabinovitch. 1997. Regulation of tenascin-C, a vascular smooth muscle cell survival factor that interacts with the $\alpha_{v} \beta_{3}$ integrin to promote epidermal growth factor receptor phosphorylation and growth. J. Cell Biol. 139:279-293.

Jones, P.L., F.S. Jones, B. Zhou, and M. Rabinovitch. 1999. Induction of vascular smooth muscle cell tenascin-C gene expression by denatured type I collagen is dependent upon a $\beta_{3}$ integrin-mediated mitogen-activated protein kinase pathway and a 122-base pair promoter element. J. Cell Sci. 112:435-445.

Jones, P.L., R. Chapados, H.S. Baldwin, G.W. Raff, E.V. Vitvitsky, T.L. Spray, and J.W. Gaynor. 2002. Altered hemodynamics controls matrix metalloproteinase activity and tenascin-C expression in neonatal pig lung. Am. J. Physiol. Lung Cell. Mol. Physiol. 282:L26-L35.

Lotz, M.M., C.A. Burdsal, H.P. Erickson, and D.R. McClay. 1989. Cell adhesion to fibronectin and tenascin: quantitative measurements of initial binding and subsequent strengthening response. J. Cell Biol. 109:1795-1805.

Mackie, E.J, R.P. Tucker, W. Halfter, R. Chiquet-Ehrismann, and H.H. Epperlein. 1988. The distribution of tenascin coincides with pathways of neural crest cell migration. Development. 102:237-250.

Matsuda, A., A. Yoshiki, Y. Tagawa, H. Matsuda, and M. Kusakabe. 1999. Corneal wound healing in tenascin knockout mouse. Invest. Ophthalmol. Vis. Sci. 40:1071-1080.

Murphy-Ullrich, J.E., V.A. Lightner, I. Aukhil, Y.Z. Yan, H.P. Erickson, and M. Hook. 1991. Focal adhesion integrity is downregulated by the alternatively spliced domain of human tenascin. J. Cell Biol. 115:1127-1136.

Norris, R.A., and M.J. Kern. 2001. Identification of domains mediating transcrip- tion activation, repression, and inhibition in the paired-related homeobox protein, Prx2 (S8). DNA Cell Biol. 20:89-99.

Owen, J.D., P.J. Ruest, D.W. Fry, and S.K. Hanks. 1999. Induced focal adhesion kinase (FAK) expression in FAK-null cells enhances cell spreading and migration requiring both auto- and activation loop phosphorylation sites and inhibits adhesion-dependent tyrosine phosphorylation of Pyk2. Mol. Cell. Biol. 19:4806-4818.

Ren, X.D., W.B. Kiosses, D.J. Sieg, C.A. Otey, D.D. Schlaepfer, and M.A. Schwartz. 2000. Focal adhesion kinase suppresses Rho activity to promote focal adhesion turnover. J. Cell Sci. 113:3673-3678.

Ridley, A.J., and A. Hall. 1992. Distinct patterns of actin organization regulated by the small GTP-binding proteins Rac and Rho. Cold Spring Harb. Symp. Quant. Biol. 57:661-671.

Saga, Y., T. Yagi, Y. Ikawa, T. Sakakura, and S. Aizawa. 1992. Mice develop normally without tenascin. Genes Dev. 6:1821-1831.

Schaller, M.D. 2001. Biochemical signals and biological responses elicited by the focal adhesion kinase. Biochim. Biophys. Acta. 1540:1-21.

Schaller, M.D., C.A. Borgman, and J.T. Parsons. 1993. Autonomous expression of a noncatalytic domain of the focal adhesion-associated protein tyrosine kinase pp125FAK. Mol. Cell. Biol. 13:785-791.

Schwartz, M.A., and M.H. Ginsberg. 2002. Networks and crosstalk: integrin signalling spreads. Nat Cell Biol. 2002 4:E65-E68.

Sieg, D.J., C.R. Hauck, and D.D. Schlaepfer. 1999. Required role of focal adhesion kinase (FAK) for integrin-stimulated cell migration. J. Cell Sci. 112: 2677-2691.

Takaishi, K., A. Kikuchi, S. Kuroda, K. Kotani, T. Sasaki, and Y. Takai. 1993. Involvement of rho p21 and its inhibitory GDP/GTP exchange protein (rho GDI) in cell motility. Mol. Cell. Biol. 13:72-79.

Tamura, M., J. Gu, E.H. Danen, T. Takino, S. Miyamoto, and K.M. Yamada. 1999. PTEN interactions with focal adhesion kinase and suppression of the extracellular matrix-dependent phosphatidylinositol 3-kinase/Akt cell survival pathway. J. Biol. Chem. 274:20693-20703.

Tucker, R.P. 2001. Abnormal neural crest cell migration after the in vivo knockdown of tenascin-C expression with morpholino antisense oligonucleotides. Dev. Dyn. 222:115-119.

Vrucinic-Filipi, N., and R. Chiquet-Ehrismann. 1993. Tenascin function and regulation of expression. Symp. Soc. Exp. Biol. 47:155-162.

Wang, H.B., M. Dembo, S.K. Hanks, and Y. Wang. 2001. Focal adhesion kinase is involved in mechanosensing during fibroblast migration. Proc. Natl. Acad. Sci. USA. 98:11295-11300.

Wenk, M.B., K.S. Midwood, and J.E. Schwarzbauer. 2000. Tenascin-C suppresses Rho activation. J. Cell Biol. 150:913-920.

Xie, B., J. Zhao, M. Kitagawa, J. Durbin, J.A. Madri, J.L. Guan, and X.Y. Fu. 2001. Focal adhesion kinase activates Stat1 in integrin-mediated cell migration and adhesion. J. Biol. Chem. 276:19512-19523. 